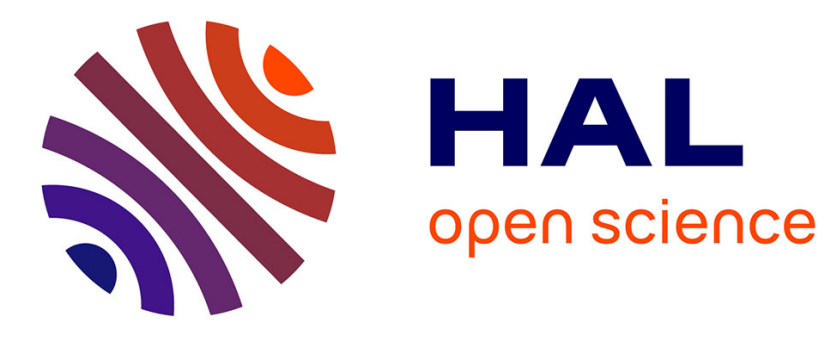

\title{
Applying relational algebra and RelView to coalition formation
}

Rudolf Berghammer, Harrie de Swart, Agnieszka Rusinowska

\section{To cite this version:}

Rudolf Berghammer, Harrie de Swart, Agnieszka Rusinowska. Applying relational algebra and RelView to coalition formation. European Journal of Operational Research, 2007, 178 (2), pp.530-542. halshs-00159845

\section{HAL Id: halshs-00159845 \\ https://shs.hal.science/halshs-00159845}

Submitted on 22 Jul 2009

HAL is a multi-disciplinary open access archive for the deposit and dissemination of scientific research documents, whether they are published or not. The documents may come from teaching and research institutions in France or abroad, or from public or private research centers.
L'archive ouverte pluridisciplinaire HAL, est destinée au dépôt et à la diffusion de documents scientifiques de niveau recherche, publiés ou non, émanant des établissements d'enseignement et de recherche français ou étrangers, des laboratoires publics ou privés. 


\title{
Applying Relational Algebra and RELVIEW to Coalition Formation ${ }^{\star}$
}

\author{
Rudolf Berghammer ${ }^{1}$, Agnieszka Rusinowska ${ }^{23}$ and Harrie de Swart ${ }^{4}$ \\ 1 Institute of Computer Science, University of Kiel \\ Olshausenstraße 40, 24098 Kiel, Germany \\ 2 Nijmegen School of Management, Radboud University Nijmegen \\ P.O. Box 9108, 6500 HK Nijmegen, The Netherlands \\ 3 Department of Mathematical Economics, Warsaw School of Economics \\ Al. Niepodleglosci 162, 02-554 Warsaw, Poland \\ 4 Department of Philosophy, Tilburg University \\ P.O. Box 90153, 5000 LE Tilburg, The Netherlands
}

\begin{abstract}
We present an application of relational algebra to coalition formation. This leads to specifications, which can be executed with the help of the RELVIEW tool after a simple translation into the tool's programming language. As an example we consider a simplification of the situation in Poland after the 2001 elections.
\end{abstract}

Keywords: RELVIEw, relational algebra, coalition formation, feasible government, dominance, stable government.

Corresponding author: Agnieszka Rusinowska.

\section{Introduction}

During the last decades a lot of coalition formation models have been proposed and investigated; see e.g., van Deemen [7] or de Vries [15] for an overview. In the present paper we focus on the model, recently introduced in Rusinowska et al. [12]. In this model, the notion of a feasible stable government is central. Roughly speaking, a feasible government is a pair consisting of a majority coalition of parties and a policy supported by this coalition. Stability of a feasible government means that it is not dominated by another feasible one. However, determining feasible stable governments can become quite complex and requires a lot of computations. This holds, in particular, if one is interested in all such governments, for example, in order to choose one of them by means of a bargaining procedure. Hence, using a computer program to calculate which governments are feasible and stable, would be extremely useful for real life applications of the model.

Since some decades relational algebra is used successfully for formal problem specification, prototyping, and algorithm development. See e.g., Schmidt et al.

\footnotetext{
* Co-operation for this paper was supported by European COST Action 274 "Theory and Applications of Relational Structures as Knowledge Instruments" (TARSKI). We are grateful to two anonymous referees for some useful suggestions.
} 
[13], Brink et al. [6], de Swart el al. [14]. Relations are well suited for modeling and reasoning about many discrete structures (like graphs, games, Petri nets, lattices) and, due to the easy mechanization (using, for instance, Boolean matrices) also for computations on them. Feasibility of a government can be described by two relations which state whether a party accepts a coalition and whether a party supports a policy. The same holds for stability. It can be defined in terms of the 'is-part-of' relations between parties and governments, the dominance relation on governments, and a list of relations comparing governments with respect to the utility of parties. Hence, relational algebra seems to be very promising for computer-aided investigations of coalition formation.

The purpose of this paper is to prove this point. We combine relational algebra and RELVIEW, a tool for the visualization and manipulation of relations and for prototyping and relational programming, to compute the set of all feasible stable governments in the sense of Rusinowska et al. [12]. To illustrate the power of the approach, we solve an example based on the real structure of the Polish government after the 2001 elections.

The remainder of the paper is organized as follows. Section 2 introduces our mathematical model of coalition formation. In Section 3 we collect the facts on relations which are necessary to treat coalition formation relation-algebraically. The treatment itself is done in Section 4 and essentially consists of the specification of the decisive notions of feasibility, dominance, and stability for governments by relation-algebraic expressions. In Section 5 we show how to translate the specifications of Section 4 into RELVIEW code and solve the Polish government example. Finally, we present some concluding remarks in Section 6 .

\section{The Mathematical Model of Coalition Formation}

In this section, we briefly recall some of the main ideas of the model of coalition formation presented in Rusinowska et al. [12], i.e., the notions essential for the application of relational algebra and ReLVIEw to the model. Let $N$ be the set of political parties, and $P$ be the set of all policies. A set of parties, i.e., an element of the powerset $2^{N}$, is called a coalition. We define a government as a pair consisting of a coalition and a policy. Hence,

$$
G:=\left\{(S, p) \mid S \in 2^{N} \wedge p \in P\right\}
$$

denotes the set of all governments. Usually, we assume that only a majority coalition (i.e., a coalition with more than half of the total number of seats in Parliament) can form a government. Nevertheless, one may easily imagine a government formed by a minority coalition.

Each party is assumed to have preferences on all policies and on all coalitions. A coalition is called feasible if it is acceptable to all its members. A policy is feasible for a given coalition if it is acceptable to all members of that coalition. A government is said to be feasible if it consists of a feasible coalition and a policy feasible for that coalition. By $G^{*}$ we denote the set of all feasible governments:

$$
G^{*}:=\{g \in G \mid G \text { is feasible }\} .
$$


For each $i \in N$, we assume a utility function $U^{(i)}: G \rightarrow \mathbb{R}$, where $U^{(i)}(g)$ denotes the utility (value) of government $g \in G$ to party $i \in N$. A precise definition of the utility of a government to a party has been given in Rusinowska et al. [12]. In Roubens et al. [10], the MacBeth technique has been applied to determine these utilities.

A feasible government $g=(S, p) \in G^{*}$ dominates a feasible government $h \in G^{*}$ (denoted as $g \succ h$ ) if the property

$$
\left(\forall i \in S: U^{(i)}(g) \geq U^{(i)}(h)\right) \wedge\left(\exists i \in S: U^{(i)}(g)>U^{(i)}(h)\right)
$$

holds. A feasible government is said to be stable if it is dominated by no feasible government. By

$$
S G^{*}:=\left\{g \in G^{*} \mid \neg \exists h \in G^{*}: h \succ g\right\}
$$

we denote the set of all (feasible) stable governments.

\section{Relational Preliminaries}

In this section, we first recall the basics of relational algebra. Next, we introduce some further relational constructions which are used in the remainder of the paper. For more details on relations and relational algebra, see e.g., Schmidt et al. [13] or Brink et al. [6].

\subsection{Relational Algebra}

If $X$ and $Y$ are sets, then a subset $R$ of the Cartesian product $X \times Y$ is called a (binary) relation with domain $X$ and range $Y$. We denote the set (in this context also called type) of all relations with domain $X$ and range $Y$ by $[X \leftrightarrow Y]$ and write $R: X \leftrightarrow Y$ instead of $R \in[X \leftrightarrow Y]$. If $X$ and $Y$ are finite sets of size $m$ and $n$ respectively, then we may consider a relation $R: X \leftrightarrow Y$ as a Boolean matrix with $m$ rows and $n$ columns. The Boolean matrix interpretation of relations is well suited for many purposes and also used as one of the graphical representations of relations within the RELVIEW tool. Therefore, in this paper we often use Boolean matrix terminology and notation. In particular, we write $R_{x, y}$ instead of $\langle x, y\rangle \in R$.

We assume the reader to be familiar with the basic operations on relations, viz. $R^{\top}$ (transposition), $\bar{R}$ (complement), $R \cup S$ (union), $R \cap S$ (intersection), $R ; S$ (composition), $R^{*}$ (reflexive-transitive closure), and the special relations $\mathrm{O}$ (empty relation), $\mathrm{L}$ (universal relation), and I (identity relation). If $R$ is included in $S$ we write $R \subseteq S$ and equality of $R$ and $S$ is denoted as $R=S$.

\subsection{Modeling of Sets}

Relational algebra offers some simple and elegant ways to describe subsets of a given set or, equivalently, predicates on this set. In this paper we will use vectors and membership-relations for this task. 
A vector $v$ is a relation $v$ with $v=v$; L. For $v$ being of type $[X \leftrightarrow Y]$ this condition means: Whatever set $Z$ and universal relation $\mathrm{L}: Y \leftrightarrow Z$ we choose, an element $x \in X$ is either in relationship $(v ; \mathrm{L})_{x, z}$ to no element $z \in Z$ or to all elements $z \in Z$. As for a vector, therefore, the range is irrelevant, we consider in the following mostly vectors $v: X \leftrightarrow \mathbf{1}$ with a specific singleton set $\mathbf{1}:=\{\perp\}$ as range and omit in such cases the second subscript, i.e., write $v_{x}$ instead of $v_{x, \perp}$.

Analogously to linear algebra we will use lower-case letters to denote vectors. A vector $v: X \leftrightarrow \mathbf{1}$ can be considered as a Boolean matrix with exactly one column, i.e., as a Boolean column vector, and describes (or: is a description of) the subset $\left\{x \in X \mid v_{x}\right\}$ of $X$.

As a second way to model sets we will use the relation-level equivalents of the set-theoretic symbol " $\in$ ", i.e., membership-relations $\varepsilon: X \leftrightarrow 2^{X}$. These specific relations are defined by $\varepsilon_{x, Y}$ if and only if $x \in Y$, for all $x \in X$ and $Y \in 2^{X}$. A Boolean matrix representation of $\varepsilon$ requires exponential space. However, in Berghammer et al. [4] an implementation of $\varepsilon$ using reduced ordered binary decision diagrams (ROBDDs) is presented, the number of vertices of which is linear in the size of $X$.

\subsection{Cartesian Products and Disjoint Unions}

Given a Cartesian product $X \times Y$ of two sets $X$ and $Y$, there are two projection functions which decompose a pair $u=\left\langle u_{1}, u_{2}\right\rangle$ into its first component $u_{1}$ and its second component $u_{2}$. For a relation-algebraic approach it is useful to consider instead of these functions the corresponding projection relations $\pi: X \times Y \leftrightarrow X$ and $\rho: X \times Y \leftrightarrow Y$ such that for all $u \in X \times Y, x \in X$, and $y \in Y$ we have $\pi_{u, x}$ if and only if $u_{1}=x$ and $\rho_{u, y}$ if and only if $u_{2}=y$. Projection relations enable us to describe the well-known pairing operation of functional programming relationalgebraically as follows: For relations $R: Z \leftrightarrow X$ and $S: Z \leftrightarrow Y$ we define their pairing (frequently also called fork or tupling) $[R, S]: Z \leftrightarrow X \times Y$ by

$$
[R, S]:=R ; \pi^{\top} \cap S ; \rho^{\top} .
$$

Using (2), for all $z \in Z$ and $u \in X \times Y$ a simple reflection shows that $[R, S]_{z, u}$ if and only if $R_{z, u_{1}}$ and $S_{z, u_{2}}$. As a consequence, the exchange relation

$$
E:=[\rho, \pi]=\rho ; \pi^{\top} \cap \pi ; \rho^{\top}
$$

of type $[X \times Y \leftrightarrow X \times Y]$ exchanges the components of a pair. This means that for all $u, v \in X \times Y$ the relationship $E_{u, v}$ holds if and only if $u_{1}=v_{2}$ and $u_{2}=v_{1}$.

Analogously to the product the disjoint union $X+Y:=(X \times\{1\}) \cup(Y \times\{2\})$ of two sets $X$ and $Y$ leads to the two injection relations $\imath: X \leftrightarrow X+Y$ and $\kappa: Y \leftrightarrow X+Y$ such that for all $u \in X+Y, x \in X$, and $y \in Y$ we have $\imath_{x, u}$ if and only if $u=\langle x, 1\rangle$ and $\kappa_{y, u}$ if and only if $u=\langle y, 2\rangle$. In this case the counterpart of pairing is the sum $R+S: X+Y \leftrightarrow Z$ of two relations $R: X \leftrightarrow Z$ and $S: Y \leftrightarrow Z$, defined by

$$
R+S:=\left(\imath^{\top} ; R\right) \cup\left(\kappa^{\top} ; S\right) .
$$


From specification (4) we obtain for all $u \in X+Y$ and $z \in Z$ that $(R+S)_{u, z}$ if and only if there exists $x \in X$ such that $u=\langle x, 1\rangle$ and $R_{x, z}$ or there exists $y \in Y$ such that $u=\langle y, 2\rangle$ and $S_{y, z}$.

We end this section with an application of the constructions introduced so far, which is used in the remainder of the paper. Our starting point is the representation of a relation $R: X \leftrightarrow Y$ by a vector $\operatorname{vec}(R): X \times Y \leftrightarrow \mathbf{1}$, which means that for all $x \in X$ and $y \in Y$ the properties $R_{x, y}$ and $\operatorname{vec}(R)_{\langle x, y\rangle, \perp}$, or $\operatorname{vec}(R)_{\langle x, y\rangle}$ for short, are equivalent. To obtain a relation-algebraic specification of $\operatorname{vec}(R)$, i.e., an expression which does not use element relationships (in our notation: does not use indices) but only the constants and operations of relational algebra, we assume $x \in X$ and $y \in Y$ and calculate as follows:

$$
\begin{array}{rlrl}
R_{x, y} & \Longleftrightarrow \exists a: \pi_{\langle x, y\rangle, a} \wedge R_{a, y} & & \pi: X \times Y \leftrightarrow X \text { projection } \\
& \Longleftrightarrow(\pi ; R)_{\langle x, y\rangle, y} & & \\
& \Longleftrightarrow \exists b:(\pi ; R)_{\langle x, y\rangle, b} \wedge \rho_{\langle x, y\rangle, b} & \rho: X \times Y \leftrightarrow Y \text { projection } \\
& \Longleftrightarrow \exists b:(\pi ; R \cap \rho)_{\langle x, y\rangle, b} \wedge \mathrm{L}_{b} & \mathrm{~L}: Y \leftrightarrow \mathbf{1} \\
& \Longleftrightarrow((\pi ; R \cap \rho) ; \mathrm{L})_{\langle x, y\rangle} . &
\end{array}
$$

An immediate consequence of the last expression of this calculation and the equality of relations is the relation-algebraic specification

$$
\operatorname{vec}(R)=(\pi ; R \cap \rho) ; \mathbf{L}
$$

of the vector $\operatorname{vec}(R): X \times Y \leftrightarrow \mathbf{1}$ (see also Schmidt et al. [13]). Finally, we consider a list $R^{(1)}, R^{(2)}, \ldots, R^{(n)}$ of relations $R^{(i)}: X \leftrightarrow Y$. Let $N:=\{1, \ldots, n\}$. If we identify this set with the disjoint union of $n$ copies of $\mathbf{1}$, then

$$
C:=\operatorname{vec}\left(R^{(1)}\right)^{\top}+\ldots+\operatorname{vec}\left(R^{(n)}\right)^{\top}
$$

defines a relation of type $[N \leftrightarrow X \times Y]$ such that, using Boolean matrix terminology, for all $i \in N$ the $i^{\text {th }}$ row of $C$ equals the transpose of the vector $\operatorname{vec}\left(R^{(i)}\right)$. Hence, from the above considerations we obtain for all $i \in N, x \in X$, and $y \in Y$ the equivalence of $C_{i,\langle x, y\rangle}$ and $R_{x, y}^{(i)}$.

\section{Relational Description of Coalition Formation}

Based on the mathematical model of coalition formation in Section 2, in this section we develop relation-algebraic specifications of feasible governments, the dominance relationship, and stable governments. As we will demonstrate in Section 5 , these can be translated immediately into the programming language of the RELVIEW tool and then applied to deal with concrete examples.

\subsection{Feasibility}

In order to develop a relation-algebraic specification of feasible governments, we need two 'acceptability' relations $A$ and $B$. We assume $A: N \leftrightarrow P$ such that

$$
A_{i, p} \Longleftrightarrow \text { party } i \text { accepts policy } p
$$


for all $i \in N$ and $p \in P$, and $B: N \leftrightarrow 2^{N}$ such that

$$
B_{i, S} \Longleftrightarrow \operatorname{party} i \text { accepts coalition } S
$$

for all $i \in N$ and $S \in 2^{N}$.

Based on the two acceptability relations $A$ and $B$ and the description of feasibility given in Section 2, next we consider the following three relations:

1. A relation isFea $(A): 2^{N} \leftrightarrow P$ such that a coalition $S \in 2^{N}$ and a policy $p \in P$ are in relationship isFea $(A)_{S, p}$ if and only if $p$ is feasible for $S$. A formal predicate logic definition of this is

$$
i s F e a(A)_{S, p} \Longleftrightarrow \forall i: i \in S \rightarrow A_{i, p} .
$$

2. A vector $f e a C(B): 2^{N} \leftrightarrow \mathbf{1}$ which describes the set of all feasible coalitions. Here we have for all $S \in 2^{N}$ the predicate logic definition

$$
f e a C(B)_{S} \Longleftrightarrow \forall i: i \in S \rightarrow B_{i, S} .
$$

3. A relation $\operatorname{fea} G(A, B): 2^{N} \leftrightarrow P$ which coincides with the set $G^{*}$ of feasible governments. Using the relations introduced so far, hence we have for all coalitions $S \in 2^{N}$ and policies $p \in P$ the description

$$
\mathrm{fea} G(A, B)_{S, p} \Longleftrightarrow \mathrm{isFea}(A)_{S, p} \wedge \mathrm{fea} C(B)_{S} .
$$

Our goal is to obtain from the predicate logic definitions (7), (8), and (9) of the relations isFea $(A), f e a C(B)$, and $f e a G(A, B)$ equivalent relation-algebraic specifications. The proof of the following theorem shows how to calculate these. Besides the predicate logic definitions and some simple logical laws it only uses well-known correspondences between certain logical constructions and relationalgebraic operations (see e.g., Schmidt et al. [13]).

Theorem 4.1 Assume $\varepsilon: N \leftrightarrow 2^{N}$ to be the membership-relation on the set of parties. Then we have

$$
\begin{gathered}
\text { isFea }(A)=\overline{\varepsilon^{\top} ; \bar{A}}, \\
f e a C(B)=\overline{(\varepsilon \cap \bar{B})^{\top} ; \mathrm{L},} \\
f e a G(A, B)=\overline{\varepsilon^{\top} ; \bar{A}} \cap \overline{(\varepsilon \cap \bar{B})^{\top} ; \mathrm{L} ; \mathrm{L} .}
\end{gathered}
$$

Proof: For all $S \in 2^{N}$ and $p \in P$ we are able to calculate

$$
\begin{aligned}
\text { isFea }_{(A)_{S, p}} & \Longleftrightarrow \forall i: i \in S \rightarrow A_{i, p} \\
& \Longleftrightarrow \neg \exists i: i \in S \wedge \bar{A}_{i, p} \\
& \Longleftrightarrow \neg \exists i: \varepsilon_{S, i}^{\top} \wedge \bar{A}_{i, p} \\
& \Longleftrightarrow\left(\overline{\varepsilon^{\top} ; \bar{A}}\right)_{S, p},
\end{aligned}
$$


and from the last expression of this derivation we get (10) due to the definition of equality of relations. In the same way (11) follows from

$$
\begin{aligned}
\text { feaC }(B)_{S} & \Longleftrightarrow \forall i: i \in S \rightarrow B_{i, S} \\
& \Longleftrightarrow \neg \exists i: i \in S \wedge \bar{B}_{i, S} \\
& \Longleftrightarrow \neg i: \varepsilon_{i, S} \wedge \bar{B}_{i, S} \\
& \Longleftrightarrow \neg \exists i:(\varepsilon \cap \bar{B})_{i, S} \\
& \Longleftrightarrow \neg \exists i:(\varepsilon \cap \bar{B})_{S, i}^{\top} \wedge \mathrm{L}_{i} \quad \mathrm{~L}: P \leftrightarrow \mathbf{1} \\
& \Longleftrightarrow\left((\varepsilon \cap \bar{B})^{\top} ; \mathrm{L}\right)_{S}
\end{aligned}
$$

for all $S \in 2^{N}$ and the equality of relations, and (12) follows from

$$
\begin{aligned}
& \text { fea } G(A, B)_{S, p} \Longleftrightarrow \text { isFea }(A)_{S, p} \wedge \text { feaC }(B)_{S} \\
& \Longleftrightarrow \text { isFea }(A)_{S, p} \wedge\left(\exists i: \text { feaC }(B)_{S, i} \wedge \mathrm{L}_{i, p}\right) \\
& \Longleftrightarrow \text { isFea }(A)_{S, p} \wedge(\text { feaC }(B) ; \mathrm{L})_{S, p} \\
& \Longleftrightarrow(\text { isFea }(A) \cap \operatorname{feaC}(B) ; \mathrm{L})_{S, p}
\end{aligned}
$$

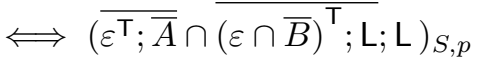

for all $S \in 2^{N}$ and $p \in P$ and the equality of relations.

It should be remarked that the relation-algebraic specification of $f e a G(A, B)$ uses two different universal relations, which are denoted with the same symbol $\mathrm{L}$. The inner $\mathrm{L}$ of $(12)$ has type $[P \leftrightarrow \mathbf{1}]$, i.e., is a universal vector, whereas the type of the outer $\mathrm{L}$ of $(12)$ is $[N \leftrightarrow P]$, i.e., this $\mathrm{L}$ is a 'proper' universal relation.

\subsection{Dominance}

Now, we consider the set $G^{*}$ of all feasible governments and want to develop a relation-algebraic specification of the dominance relationship between feasible governments. To this end, we suppose a relational description of government membership to be given, that is, a relation $M: N \leftrightarrow G^{*}$ such that

$$
M_{i, g} \Longleftrightarrow \operatorname{party} i \text { is a member of government } g
$$

for all $i \in N$ and $g \in G^{*}$. Moreover, for each party $i \in N$, we introduce a utility (or comparison) relation $R^{(i)}: G^{*} \leftrightarrow G^{*}$ by the definition

$$
R_{g, h}^{(i)} \Longleftrightarrow U^{(i)}(g) \geq U^{(i)}(h)
$$

for all $g, h \in G^{*}$. Based on these relations, we are able to introduce a global utility (or comparison) relation $C: N \leftrightarrow G^{*} \times G^{*}$ as follows: For all $i \in N$ and $g, h \in G^{*}$ we define $C_{i,\langle g, h\rangle}$ if and only if $R_{g, h}^{(i)}$. If the set $N$ is finite and enumerated, i.e., $N=\left\{i_{1}, \ldots, i_{n}\right\}$, then this leads to the list $R^{\left(i_{1}\right)}, \ldots, R^{\left(i_{n}\right)}$ of utility relations and using (5) and (6) we immediately get the relation-algebraic specification

$$
C=\operatorname{vec}\left(R^{\left(i_{1}\right)}\right)^{\top}+\ldots+\operatorname{vec}\left(R^{\left(i_{n}\right)}\right)^{\top} .
$$


After these preparations, now we consider the dominance relationship. Combining its predicate logic definition in (1) with the relations $M$ and $C$, we get

$$
g \succ h \Longleftrightarrow\left(\forall i: M_{i, g} \rightarrow C_{i,\langle g, h\rangle}\right) \wedge\left(\exists i: M_{i, g} \wedge \bar{C}_{i,\langle h, g\rangle}\right)
$$

for all $g, h \in G^{*}$. Now, we use the exchange relation $E: G^{*} \times G^{*} \leftrightarrow G^{*} \times G^{*}$ and transform the expression $\bar{C}_{i,\langle h, g\rangle}$ of (14) as follows:

$$
\begin{aligned}
\bar{C}_{i,\langle h, g\rangle} & \Longleftrightarrow \exists\langle a, b\rangle: \bar{C}_{i,\langle a, b\rangle} \wedge\langle a, b\rangle=\langle h, g\rangle \\
& \Longleftrightarrow \exists\langle a, b\rangle: \bar{C}_{i,\langle a, b\rangle} \wedge E_{\langle a, b\rangle,\langle g, h\rangle} \\
& \Longleftrightarrow(\bar{C} ; E)_{i,\langle g, h\rangle} .
\end{aligned}
$$

This equivalence yields the following description of dominance:

$$
g \succ h \Longleftrightarrow\left(\forall i: M_{i, g} \rightarrow C_{i,\langle g, h\rangle}\right) \wedge\left(\exists i: M_{i, g} \wedge(\bar{C} ; E)_{i,\langle g, h\rangle}\right) .
$$

The use of pairs $\langle g, h\rangle$ and the quantified $i$ in the right-hand side of (15) suggests to specify the dominance relationship as a vector dominance $(M, C): G^{*} \times G^{*} \leftrightarrow \mathbf{1}$ which describes the set of pairs $u \in G^{*} \times G^{*}$ such that the first component dominates the second component. In the proof of the next theorem we show how to obtain from (15) a relation-algebraic specification of this vector.

Theorem 4.2 Let $\pi: G^{*} \times G^{*} \leftrightarrow G^{*}$ and $\rho: G^{*} \times G^{*} \leftrightarrow G^{*}$ be the projection relations and $E: G^{*} \times G^{*} \leftrightarrow G^{*} \times G^{*}$ the exchange relation. If we define

$$
\text { dominance }(M, C)=\overline{\left(\pi ; M^{\top} \cap \bar{C}^{\top}\right) ; \mathrm{L}} \cap\left(\pi ; M^{\top} \cap E ; \bar{C}^{\top}\right) ; \mathrm{L},
$$

then we have for all $u=\langle g, h\rangle \in G^{*} \times G^{*}$ that dominance $(M, C)_{u}$ if and only if $g \succ h$.

PROOF: We start our calculation with the first conjunct of the right-hand side of (15) and transform it as follows:

$$
\begin{aligned}
\forall i: M_{i, g} \rightarrow C_{i,\langle g, h\rangle} & \Longleftrightarrow \neg \exists i: M_{i, g} \wedge \bar{C}_{i,\langle g, h\rangle} \\
& \Longleftrightarrow \neg \exists i: M_{g, i}^{\top} \wedge \bar{C}_{i, u} \\
& \Longleftrightarrow \neg \exists i:\left(\exists g^{\prime}: \pi_{u, g^{\prime}} \wedge M_{g^{\prime}, i}^{\top}\right) \wedge \bar{C}_{u, i}^{\top} \\
& \Longleftrightarrow \neg \exists i:\left(\pi ; M^{\top}\right)_{u, i} \wedge \bar{C}_{u, i} \\
& \Longleftrightarrow \neg \exists i:\left(\pi ; M^{\top} \cap \bar{C}^{\top}\right)_{u, i} \wedge \mathrm{L}_{i} \quad \mathrm{~L}: N \leftrightarrow \mathbf{1} \\
& \Longleftrightarrow\left(\left(\pi ; M^{\top} \cap \bar{C}^{\top}\right) ; \mathrm{L}\right)_{u} .
\end{aligned}
$$

In a similar way we treat the second conjunct of the right-hand side of (15). Here we can proceed as follows:

$$
\begin{array}{rlrl}
\exists i: M_{i, g} \wedge(\bar{C} ; E)_{i,\langle g, h\rangle} & \Longleftrightarrow \exists i:\left(\pi ; M^{\top}\right)_{u, i} \wedge(\bar{C} ; E)_{i,\langle g, h\rangle} & & \text { see above } \\
& \Longleftrightarrow \exists i:\left(\pi ; M^{\top}\right)_{u, i} \wedge(\bar{C} ; E)_{u, i}^{\mathrm{T}} \wedge \mathrm{L}_{i} & \mathrm{~L}: N \leftrightarrow \mathbf{1} \\
& \Longleftrightarrow \exists i:\left(\pi ; M^{\top}\right)_{u, i} \wedge\left(E ; \bar{C}^{\mathrm{T}}\right)_{u, i} \wedge \mathrm{L}_{i} & & E=E^{\mathrm{\top}} \\
& \Longleftrightarrow \exists i:\left(\pi ; M^{\top} \cap E ; \bar{C}^{\mathrm{T}}\right)_{u, i} \wedge \mathrm{L}_{i} & \\
& \Longleftrightarrow\left(\left(\pi ; M^{\top} \cap E ; \bar{C}^{\mathrm{\top}}\right) ; \mathrm{L}\right)_{u} . &
\end{array}
$$


Combining these derivations with the definition (16) of dominance $(M, C)$ and property (15) yields the desired result.

During the proof of Theorem 4.2 we have applied the symmetry of the exchange relation $E$ to make a later RELVIEW-implementation of dominance $(M, C)$ more efficient. Symmetry of $E$ is an immediate consequence of its element-wise description. But it also can be shown with the help of the relation-algebraic specification (3) of $E$ and a relation-algebraic axiomatization of the projection relations as, e.g., presented in Schmidt et al. [13].

If one is interested in the dominance relationship between feasible governments as a relation of type $\left[G^{*} \leftrightarrow G^{*}\right]$ instead of a vector as we described it, then such a relation can be obtained using that $\operatorname{rel}(v)=\pi^{\top} ;(\rho \cap(v ; \mathrm{L}))$ describes a vector $v: X \times Y \leftrightarrow \mathbf{1}$ as relation $\operatorname{rel}(v): X \leftrightarrow Y$, i.e., $v_{\langle x, y\rangle}$ and $\operatorname{rel}(v)_{x, y}$ are equivalent for all $x \in X$ and $y \in Y$ (where $\pi, \rho$ are the projection relations; see again Schmidt et al. [13]).

\subsection{Stability}

Finally, we consider stability of feasible governments. Here we start with a stability vector stable $(M, C): G^{*} \leftrightarrow \mathbf{1}$ which describes the subset $S G^{*}$ of $G^{*}$, i.e., the subset of all (feasible) stable governments. From the definition of $S G^{*}$ in Section 2 and Theorem 4.2 we get

$$
\operatorname{stable}(M, C)_{g} \Longleftrightarrow \neg \exists h: \operatorname{dominance}(M, C)_{\langle h, g\rangle}
$$

for all $g \in G^{*}$. This description immediately yields a relation-algebraic specification of stability.

Theorem 4.3 Let $\rho: G^{*} \times G^{*} \leftrightarrow G^{*}$ be the second projection relation of the Cartesian product $G^{*} \times G^{*}$. Then

$$
\operatorname{stable}(M, C)=\overline{\rho^{\top} ; \operatorname{dominance}(M, C)} .
$$

Proof: For all $g \in G^{*}$ we have

$$
\begin{aligned}
\operatorname{stable}(M, C)_{g} & \Longleftrightarrow \neg \exists h: \text { dominance }(M, C)_{\langle h, g\rangle} \\
& \Longleftrightarrow \neg \exists u: \rho_{u, g} \wedge \text { dominance }(M, C)_{u} \\
& \Longleftrightarrow \neg \exists u: \rho_{g, u}^{\top} \wedge \text { dominance }(M, C)_{u}
\end{aligned}
$$

and, hence, (18) is a consequence of the equality of relations.

\section{Implementation and Example}

In order to illustrate our approach, in the following we use the RELVIEW tool to solve a simple theoretical example based on the real structure of the Polish government after the 2001 elections. The extended version of this example, without using ReLVIEw, is presented in Rusinowska et al. [11]. 


\subsection{The RelView Tool}

Relational algebra has a fixed and surprisingly small set of constants and operations which - in the case of finite carrier sets - can be implemented very efficiently. At Kiel University we have developed a visual computer system for the visualization and manipulation of relations and for relational prototyping and programming, called RELVIEW. The tool is written in the C programming language, uses reduced ordered binary decision diagrams for implementing relations, and makes full use of the X-windows graphical user interface. Details and applications can be found, for instance, in Berghammer et al. [3], Behnke et al. [1], Berghammer et al. [4], and Berghammer et al. [5].

The main purpose of the RELVIEW tool is the evaluation of relation-algebraic expressions. These are constructed from the relations of its workspace using predefined operations and tests, user-defined relational functions, and user-defined relational programs. A relational program is much like a function procedure in the programming languages Pascal or Modula 2, except that it only uses relations as data type. It starts with a head line containing the program name and the list of formal parameters, which stand for relations. Then the declaration of the local relational domains, functions, and variables follows. Domain declarations can be used to introduce projection relations and pairings of relations in the case of Cartesian products, and injection relations and sums of relations in the case of disjoint unions, respectively. The third part of a program is the body, a while-program over relations. As a program computes a value, finally, its last part consists of a return-clause, which is a relation-algebraic expression whose value after the execution of the body is the result.

For example, the relation-algebraic specification dominance $(M, C)$ of the vector describing the dominance relationship between feasible governments immediately leads to the following RELVIEW-program.

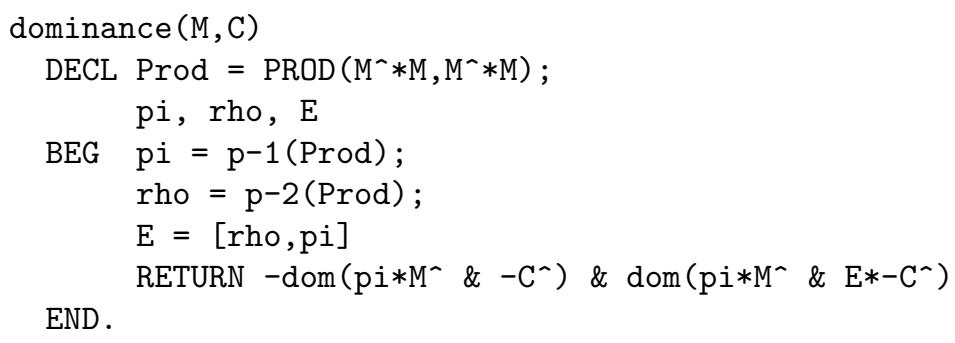

In this program the first declaration introduces Prod as a name for the direct product $G^{*} \times G^{*}$. Using Prod, the projection relations and the exchange relation are then computed by the three assignments of the body and stored as pi, rho, and E, respectively. The return-clause of the program consists of a direct translation of (16) into RELVIEW-notation, where - , -, \&, and * denote transposition, complement, intersection, and composition, and, furthermore, the pre-defined operation dom computes for a relation $R: X \leftrightarrow Y$ the vector $R$; $\mathrm{L}: X \leftrightarrow \mathbf{1}$.

Also a translation of the remaining relation-algebraic specifications of Section 4 into RELVIEW-code is straightforward. 


\subsection{The Example}

In order to illustrate the application of RELVIEW to coalition formation, we use a theoretical example presented in Rusinowska et al. [11]. It is based on the structure of Polish Parliament after the 2001 elections. The Lower House of Polish Parliament after these elections consists of seven parties: the Alliance of the Democratic Left and Labor Union (SLD), Civil Platform (PO), Self-Defence, Law and Justice (PiS), Polish Peasant Party (PSL), Polish Family Alliance, and German Minority.

In our example, the acceptability relationships showed that 17 governments are feasible. Only four of the seven parties are involved in these feasible governments, viz. SLD, PO, PiS, and PSL. The following picture shows the membership relation $M$ as Boolean $4 \times 17$ matrix as presented by RELVIEW. Here a black square means 1 and a white square means 0 so that, for example, the first government consists of SLD and PO.

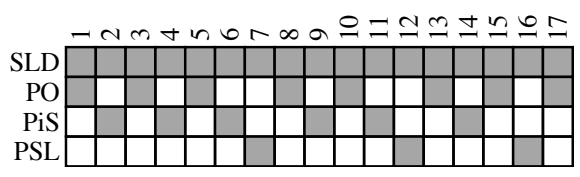

Based on the parties' preferences, in Rusinowska et al. [11] the following utilities of the feasible governments have been determined.

\begin{tabular}{|c||c|c|c|c|}
\hline Government & $U^{(\mathrm{SLD})}(g)$ & $U^{(\mathrm{PO})}(g)$ & $U^{(\mathrm{PiS})}(g)$ & $U^{(\mathrm{PSL})}(g)$ \\
\hline \hline$g_{1}=\left(\mathrm{SLD}-\mathrm{PO}, p_{1}\right)$ & $\frac{4}{3}$ & $\frac{7}{3}$ & $\frac{4}{3}$ & $-\frac{7}{3}$ \\
\hline$g_{2}=\left(\mathrm{SLD}-\mathrm{PiS}, p_{1}\right)$ & $\frac{2}{3}$ & -1 & $\frac{5}{3}$ & $-\frac{17}{3}$ \\
\hline$g_{3}=\left(\mathrm{SLD}-\mathrm{PO}, p_{2}\right)$ & 2 & $\frac{5}{3}$ & 2 & $-\frac{17}{3}$ \\
\hline$g_{4}=\left(\mathrm{SLD}-\mathrm{PiS}, p_{2}\right)$ & $\frac{4}{3}$ & $-\frac{5}{3}$ & $\frac{7}{3}$ & -9 \\
\hline$g_{5}=\left(\mathrm{SLD}-\mathrm{PO}, p_{3}\right)$ & $\frac{4}{3}$ & $\frac{7}{3}$ & $\frac{4}{3}$ & $\frac{4}{3}$ \\
\hline$g_{6}=\left(\mathrm{SLD}-\mathrm{PiS}, p_{3}\right)$ & $\frac{2}{3}$ & -1 & $\frac{5}{3}$ & -2 \\
\hline$g_{7}=\left(\mathrm{SLD}-\mathrm{PSL}, p_{3}\right)$ & $\frac{5}{3}$ & 2 & $\frac{4}{3}$ & 2 \\
\hline$g_{8}=\left(\mathrm{SLD}-\mathrm{PO}, p_{4}\right)$ & 2 & $\frac{5}{3}$ & 2 & -2 \\
\hline$g_{9}=\left(\mathrm{SLD}-\mathrm{PiS}, p_{4}\right)$ & $\frac{4}{3}$ & $-\frac{5}{3}$ & $\frac{7}{3}$ & $-\frac{16}{3}$ \\
\hline$g_{10}=\left(\mathrm{SLD}-\mathrm{PO}, p_{5}\right)$ & $\frac{5}{3}$ & $\frac{7}{3}$ & $\frac{4}{3}$ & $\frac{4}{3}$ \\
\hline$g_{11}=\left(\mathrm{SLD}-\mathrm{PiS}, p_{5}\right)$ & 1 & -1 & $\frac{5}{3}$ & -2 \\
\hline$g_{12}=\left(\mathrm{SLD}-\mathrm{PSL}, p_{5}\right)$ & 2 & 2 & $\frac{4}{3}$ & 2 \\
\hline$g_{13}=\left(\mathrm{SLD}-\mathrm{PO}, p_{6}\right)$ & $\frac{7}{3}$ & $\frac{5}{3}$ & 2 & -2 \\
\hline$g_{14}=\left(\mathrm{SLD}-\mathrm{PiS}, p_{6}\right)$ & $\frac{5}{3}$ & $-\frac{5}{3}$ & $\frac{7}{3}$ & $-\frac{16}{3}$ \\
\hline$g_{15}=\left(\mathrm{SLD}-\mathrm{PO}, p_{7}\right)$ & 2 & 2 & $-\frac{8}{3}$ & $\frac{2}{3}$ \\
\hline$g_{16}=\left(\mathrm{SLD}-\mathrm{PSL}, p_{7}\right)$ & $\frac{7}{3}$ & $\frac{5}{3}$ & $-\frac{8}{3}$ & $\frac{4}{3}$ \\
\hline$g_{17}=\left(\mathrm{SLD}-\mathrm{PO}, p_{8}\right)$ & $\frac{8}{3}$ & $\frac{4}{3}$ & -2 & $-\frac{8}{3}$ \\
\hline
\end{tabular}


With the help of the data presented in this table we can define the utility relations $R^{(i)}$, where $i \in\{\mathrm{SLD}, \mathrm{PO}, \mathrm{PiS}, \mathrm{PSL}\}$. To give an impression how these relations look as RELVIEW-matrices, the next two pictures show the matrices for $R^{\text {(SLD) }}$ (left-hand side) and $R^{(\mathrm{PO})}$ (right-hand side).
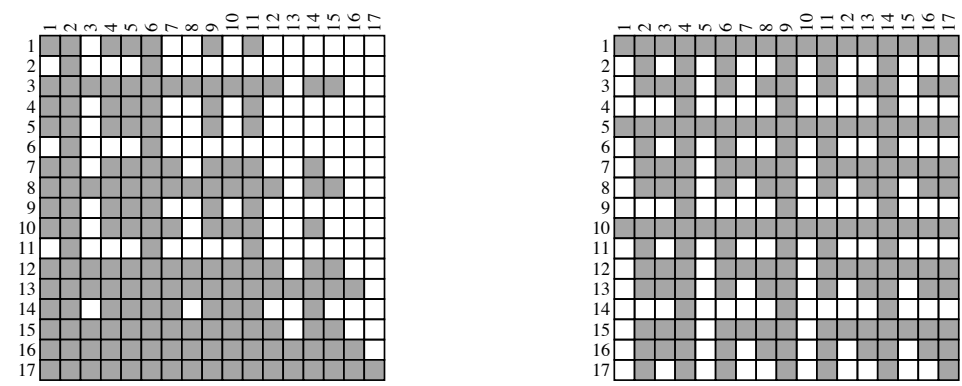

An easy way to obtain these relations is, first to draw - from the table above the directed graphs expressing the immediate neighborhood relationships of the utilities, and then to compute the reflexive-transitive closures of the graphs' relations. For example, the above matrices are obtained from the following graphs:
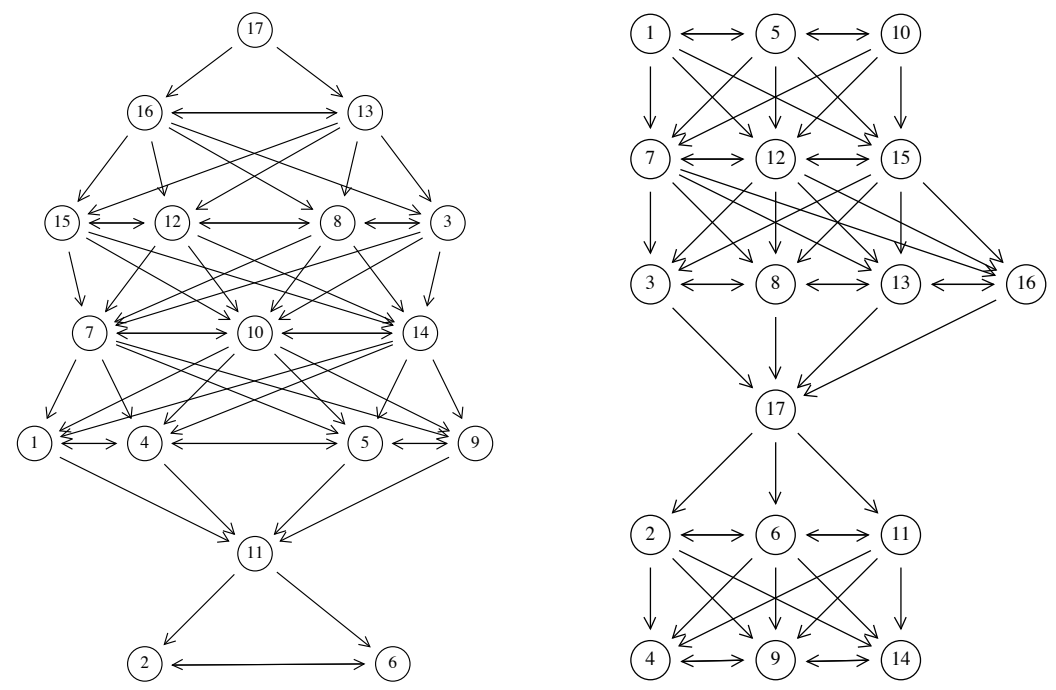

At this place we would like to point out that theoretically it is not necessary that the utilities of all governments to the parties are available as real numbers. As the two pictures show, it would suffice to have the relationships at hand which are expressed by the different horizontal layers of the graphs of the relations $R^{(i)}$ and, e.g., obtained by a finite and linearly ordered set of utilities. But the techniques and systems used in practice (e.g., MacBeth in Roubens et al. [10]) usually assume numerical values for all utilities.

Having defined the membership relation and the utility (or comparison) relations, the RELVIEW system is able to compute the global utility relation $C$ 
and, after that, the vector describing the set of stable governments. The output of our example, i.e., the vector showing all stable governments, is presented in the following picture. To save place, it shows the vector stable $(M, C)$ in the transposed form, i.e., as Boolean $1 \times 17$ matrix.

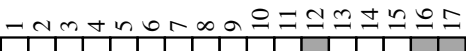

According to this Boolean row vector, 3 out of 17 feasible governments are stable: governments $g_{12}, g_{16}$ and $g_{17}$. We like to point out that it took only 0.04 second for RELVIEW to determine these stable governments on a Sun-Fire 880 workstation (running Solaris 9 at $750 \mathrm{MHz}$ ).

To test the efficiency of our RELVIEW-programs, we have performed further experiments with randomly generated larger examples, e.g., 20 parties and 400 feasible governments. In each case it took at most some seconds to compute all stable governments - although 400 feasible governments lead to a $C$-matrix with 160000 columns.

If there are (as in the Polish elections example) only a small number of feasible governments, then a RELVIEW-user immediately obtains the stable governments from their vector description or, as an alternative, by first computing dominance as a relation on $G^{*} \times G^{*}$ as sketched in Section 4.2 (applying the operation rel to the vector dominance $(M, C)$ ) and looking after that for the sources (roots) of the graph-representation of this relation. In the latter case, things become even more easy if one considers only the graph of the immediate neighborhood relationships of the dominance relation, that is, a so-called transitive reduction. For the Polish government example, we obtain the following picture (drawn with RELVIEw using the layout algorithm of Gansner et al. [8]), showing again that $g_{12}, g_{16}$ and $g_{17}$ are the stable governments:

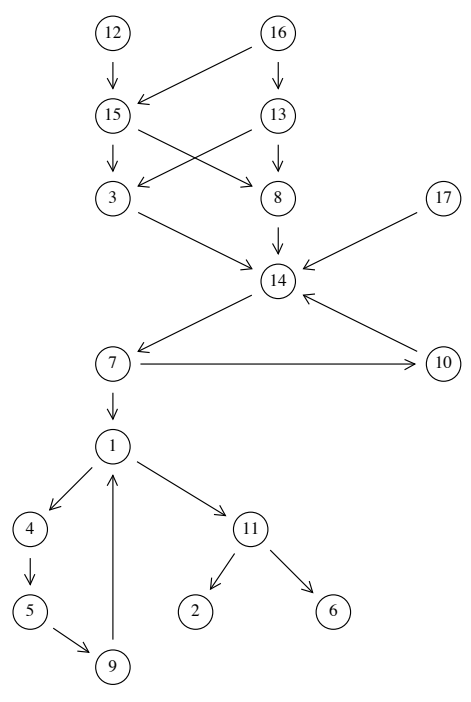


In the case of a large number of feasible governments, say 400 as mentioned above, stability hardly can be seen from the vector or the graph description. Nevertheless, there is a very understandable RELVIEW-visualization of the set of all stable governments, viz. as the columns of a relation of type $\left[M \leftrightarrow S G^{*}\right]$. In our example, this relation looks as given in the following picture:

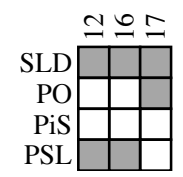

The general method to obtain such a column-wise description of stable governments is to compute the composition $M ; i d^{\top}$, where $M: N \leftrightarrow G^{*}$ is the relation describing government membership and $i d: S G^{*} \leftrightarrow G^{*}$ is the representation of the identical function from $S G^{*}$ to $G^{*}$ as a relation. The computation of $i d$ with the RELVIEW tool is very easy (and fast), since this relation corresponds to the so-called injective mapping generated by the vector stable $(M, C)$ and RELVIEW possesses a corresponding pre-defined operation inj for computing such mappings from vectors. A formal relation-algebraic specification of this operation, e.g., can be found in Berghammer et al. [2].

\section{Conclusion}

We formulated the notions of feasibility, dominance, and stability for governments in relation-algebraic terms. This enables us to use the RELVIEW tool to compute which governments are stable. Such an application is important if one wants to apply the coalition formation model of Rusinowska et al. [12] to real life situations. We illustrated the use of RELVIEW by a simplified version of the formation of a government in Poland after the 2001 elections.

We feel that our approach has some advantages. First of all, the formality of relational algebra drastically reduces the danger of making errors. As our examples (and many others) show, it is often possible to 'calculate' concise relational algorithms from formal mathematical specifications, so that correctness is guaranteed by construction. Secondly, the application of RELVIEW not only allows a relational algorithm to be executed after its simple translation into the system's programming language. RELVIEW also can be used to visualize its results and to animate its mode of operation. Besides its efficiency (which follows from the fact that the underlying technology is based on ROBDDs), we believe that the real attraction of the tool is its flexibility and the shortness and clearness of its programs (a consequence of relational algebra). New relation-based concepts are introduced all the time - in decision theory as well as in other disciplines - and experience has taught us that RELVIEW is an ideal tool for experimenting and playing with them while avoiding unnecessary overhead. This is demonstrated by many other examples, including also game-theoretic ones (like the computation of all kernels and winning positions if a two-player game is described by a graph; see e.g., von Karger and Berghammer [9] and Berghammer et al. [2]). 
For the future we plan to develop relation-algebraic formulations of bargaining procedures, in order to be able to select a specific stable government if more than one stable government exists. We are also interested in applications of relational algebra and the RELVIEW tool to other problems of decision theory, for instance choices in social groups where the decision of group members can be influenced by other ones.

\section{References}

1. R. Behnke, R. Berghammer, E. Meyer and P. Schneider, RelView - A system for calculation with relations and relational programming, In: E. Astesiano (ed.), Proc. Conf. "Fundamental Approaches to Software Engineering (FASE '98)", LNCS 1382, Springer, 318-321 (1998)

2. R. Berghammer and T. Hoffmann, Deriving relational programs for computing kernels by reconstructing a proof of Richardson's theorem, Science of Computer Programming 38, 1-25 (2000)

3. R. Berghammer, B. Karger von and C. Ulke, Relation-algebraic analysis of Petri nets with ReLVIEw, In: T. Margaria and B. Steffen (eds.), Proc. 2nd Workshop "Tools and Applications for the Construction and Analysis of Systems (TACAS '96)", LNCS 1055, Springer, 49-69 (1996)

4. R. Berghammer, B. Leoniuk and U. Milanese, Implementation of relational algebra using binary decision diagrams, In: H. Swart de (ed.), Proc. 6th Int. Workshop "Relational Methods in Computer Science", LNCS 2561, Springer, 241-257 (2002)

5. R. Berghammer, G. Schmidt and M. Winter, RELVIEW and RATH - Two systems for dealing with relations, In: [14], 1-16 (2003)

6. C. Brink, W. Kahl and G. Schmidt (eds.), Relational Methods in Computer Science, Advances in Computing Science, Springer (1997)

7. A. Deemen van, Coalition formation and social choice, Kluwer (1997)

8. E.R. Gansner, E. Koutsofios, S.C. North and K.P. Vo, A technique for drawing directed graphs, IEEE Trans. Software Eng. 19, 214-230 (1993).

9. B. Karger von and R. Berghammer, Computing kernels in directed bichromatic graphs, Information Processing Letters 62, 5-11 (1997)

10. M. Roubens, A. Rusinowska and H. Swart de, Using MacBeth to determine utilities of governments to parties in coalition formation, Forthcoming in European Journal of Operational Research (2005)

11. A. Rusinowska and H. Swart de, Negotiating a stable government - an application of bargaining theory to a coalition formation model, Submitted (2004)

12. A. Rusinowska, H. Swart de and J.W. Rijt van der, A new model of coalition formation, Social Choice and Welfare 24, 129-154 (2005)

13. G. Schmidt and T. Ströhlein, Relations and Graphs, Discrete Mathematics for Computer Scientists, EATCS Monographs on Theoret. Comput. Sci., Springer (1993)

14. H. Swart de, E. Orlowska, G. Schmidt and M. Roubens (eds.), Theory and Applications of Relational Structures as Knowledge Instruments, LNCS 2929, Springer (2003)

15. M. Vries de, Covering with your closest neighbour: An assessment of spatial coalition formation theories, Print Partners Ipskamp (1999) 